\title{
An innovative panel to assess endothelial integrity of pedicled and skeletonized internal thoracic artery used as aortocoronary bypass graft: a randomized comparative histologic and immunohistochemical study
}

\author{
Mateusz Puslecki ${ }^{1,2}$, Piotr Buczkowski ${ }^{2}$, Michał Nowicki ${ }^{3}$, Patrycja Sujka-Kordowska ${ }^{3}$, Marcin Ligowski ${ }^{2}$, \\ Marcin Misterski ${ }^{2}$, Sebastian Stefaniak ${ }^{2}$, Marek Dąbrowski ${ }^{1}$, Agata Dabrowska ${ }^{1}$, Michal Bocianski ${ }^{2}$, \\ Lukasz Szarpak ${ }^{4}$, Kurt Ruetzler ${ }^{5}$, Marek Jemielity ${ }^{2}$, Bartlomiej Perek ${ }^{2}$ \\ ${ }^{1}$ Department of Medical Rescue, ${ }^{2}$ Department of Cardiac Surgery and Transplantology, ${ }^{3}$ Department of Histology and Embryology, Poznan \\ University of Medical Sciences, Poznan, Poland; ${ }^{4}$ Lazarski University, Warsaw, Poland; ${ }^{5}$ Department of General Anesthesiology, Anesthesiology \\ Institute, Cleveland Clinic, Cleveland, Ohio, USA \\ Contributions: (I) Conception and design: M Puslecki, B Perek; (II) Administrative support: M Ligowski, M Misterski; (III) Provision of study \\ materials or patients: M Nowicki, P Sujka-Kordowska; (IV) Collection and assembly of data: All authors; (V) Data analysis and interpretation: M \\ Puslecki, S Stefaniak, M Dąbrowski, M Ligowski, L Szarpak; (VI) Manuscript writing: All authors; (VII) Final approval of manuscript: All authors. \\ Correspondence to: Lukasz Szarpak. Lazarski University, 43 Swieradowska Str., 02-662 Warsaw, Poland. Email: lukasz.szarpak@gmail.com.
}

\begin{abstract}
Background: Optimal preservation of endothelial integrity of the vessels used as aortocoronary grafts is a crucial determinant of long-term clinical success of coronary artery bypass grafting (CABG). The purpose of this study was to evaluate an impact of two common techniques to harvest left internal thoracic artery (LITA) on endothelial integrity.

Methods: One hundred twenty consecutive patients ( 84 males and 36 females) with a mean age of $64.9 \pm 8.8$ years undergoing $\mathrm{CABG}$ were randomized to receive pedicled (group $\mathrm{P} ; \mathrm{n}=60$ ) or skeletonized (group S; n=60) LITA grafts. During surgery LITA was harvested by the same experienced cardiac surgeon. The most peripheral surplus segments of LITA were obtained and then analysed histologically under light microscope. Additionally, endothelial expression of CD31, CD34, CD133 and nitric oxide synthase (eNOS) were evaluated by means of immunohistochemistry.

Results: In both groups, no cases of major arterial wall damage such as disruption, dissection, thrombosis or subadventitial hematoma were noted on LITA cross sections. Immunohistochemical assessment of protein expression revealed no differences in endothelial expression of CD133, CD34 antigens (markers of regeneration potential) and eNOS (indicating preserved functional integrity) between studied groups. Contrary to them, endothelial immunoreactivity of CD31, a marker of the morphological integrity of the endothelium, was revealed to be stronger in group P.

Conclusions: The skeletonized method of LITA harvesting may be associated with worse preservation of morphological integrity of endothelium but without compromising functional integrity and potential for tissue regeneration.
\end{abstract}

Keywords: Aortocoronary bypass grafts; internal thoracic artery; pedicled graft; skeletonized artery; endothelial integrity; immunohistochemistry

Submitted Apr 22, 2018. Accepted for publication Jul 12, 2018.

doi: $10.21037 /$ jtd.2018.07.62

View this article at: http://dx.doi.org/10.21037/jtd.2018.07.62 


\section{Introduction}

There are any clear evidences left internal thoracic artery (LITA) is the most optimal natural conduit to bypass severely stenotic or occluded segments of the coronary arteries during coronary artery bypass grafting (CABG) (1-3). Two different techniques to harvest this artery have been applied (4). However, cardiac surgeons still debate which method is the most appropriate in the terms of surgical revascularization success (5). Clinically, it is defined as symptom-free followup period whereas angiographically as long-term graft patency $(1,6)$. Without any doubts, an application of skeletonized technique of LITA harvesting during CABG enables to obtain longer graft with higher resting blood outflow. It is also associated with less trauma to the wall chest that results in better wound healing that is of paramount importance in high risk patients (e.g., diabetic) (7). This method is also associated with marked reduction in late postoperative pain intensity and sensory disturbances in comparison with classical pedicle graft technique (8). However, up to now it has not been established which technique of LITA harvesting, pedicled or skeletonized is less traumatic for the wall integrity that could be rationale to estimated late (at least 10-15 years after surgery) clinical CABG results. Contrary to LITA, such study was carried out for the saphenous vein (SV) grafts. The authors concluded that there were clear enhancements in vessel wall properties at a cellular level and angiographical evidence of superior graft patency when the no-touch SV harvesting technique was employed (9). Thus, some researchers have expressed concern about the possible detrimental effect of skeletonization on the LITA endothelial integrity and function (10). Morphological and functional integrity of endothelium may be assessed on the base of tissue expression of specific proteins. Analyses of endothelial expressions of CD31 and endothelial nitric oxide synthase (eNOS), respectively the markers of morphological and functional integrity, were found to be useful in evaluation of perioperative microinjury of the other vessels applied as aortocoronary grafts $(11,12)$. The other proteins expression, like CD34 and CD133 typically observed in the circulating endothelial progenitor cells (EPCs), may define potential for vascular repair and restoration of normal endothelial function $(13,14)$.

In view of the above, the purpose of this study was to evaluate an impact of two different harvesting techniques of LITA—skeletonization and pedicled-on functional and morphological integrity of the endothelium assessed on the base of immunoreactivity analysis of a novel panel of proteins.

\section{Methods}

\section{Patients}

One hundred twenty consecutive patients (84 males and 36 females) with a mean age of $64.9 \pm 8.8$ years undergoing $\mathrm{CABG}$ were randomly assigned to receive a pedicled (group $\mathrm{P} ; \mathrm{n}=60$ ) or skeletonized (group $\mathrm{S} ; \mathrm{n}=60$ ) LITA grafts. The study protocol was approved by the local bioethical committee and all study participants expressed informed consent to randomization and specimen collection. The most important preoperative data are outlined in Table 1.

\section{Surgical technique}

All LITA grafts were obtained by this same experienced surgeon, who used the same operative technique and who was blinded to the aim of the study. After operations were carried out from median sternotomy. If the LITA was harvested as a pedicled conduit, a longitudinal incision of the internal thoracic fascia, parallel and medial to the medial mammary vein, was performed along the course of the artery; after blunt dissection the intercostal and sternal LITA branches were clipped distally and transected using a monopolar electrocautery $(40 \mathrm{~W})$ (Force FX-Valleylab, Covidien, USA). Eventually, the artery with its pedicle was isolated from the origin to the epigastric bifurcation. When the LITA was isolated in a skeletonized technique, the longitudinal incision of the fascia was performed between the artery and the medial concomitant vein. This incision was carried out with very low voltage electrocautery $(20 \mathrm{~W})$ to avoid any thermal damage to the arterial wall. The artery was isolated from any other surrounding structure. Only harvested LITA grafts with outflow exceeding $40 \mathrm{~mL} / \mathrm{min}$ were considered as suitable for both coronary arteries grafting and research studies. The most proximal surplus segments of LITA, minimum $5 \mathrm{~mm}$ in length, were obtained for further histological examinations.

\section{Microscopic evaluation}

All specimens soon after harvesting were fixed in Bouin's solution at room temperature (RT) for 12-24 hours, depending on thickness. Then they were transferred to the Department of Histology, rinsed in phosphate buffered 
Table 1 Preoperative characteristics and risk factors for coronary artery disease development

\begin{tabular}{|c|c|c|c|}
\hline Parameter & Group P (received pedicled grafts) $(n=60)$ & Group S (received skeletonized grafts) $(n=60)$ & $\mathrm{P}$ \\
\hline Gender, n (\%) & & & NS \\
\hline Female & $22(36.7)$ & $14(23.3)$ & \\
\hline Male & $38(63.3)$ & $46(76.7)$ & \\
\hline Weight (kg) & $78.0 \pm 13.7$ & $83.5 \pm 16.4$ & NS \\
\hline BMI $\left(\mathrm{kg} / \mathrm{m}^{2}\right)$ & $27.2(22.4-34.9)$ & $29.4(20.4-34.9)$ & NS \\
\hline EuroSCORE II logistic (\%) & $1.87(0.88-15.40)$ & $2.26(0.88-6.75)$ & NS \\
\hline NYHA, n (\%) & & & NS \\
\hline III & $0(0)$ & $6(10.0)$ & \\
\hline Previous coronary events, $\mathrm{n}(\%)$ & $26(43.3)$ & $32(53.3)$ & NS \\
\hline Diabetes mellitus, n (\%) & $18(30.0)$ & $16(26.7)$ & NS \\
\hline Arterial hypertension, n (\%) & $42(70.0)$ & $44(73.3)$ & NS \\
\hline Dyslipidaemia, n (\%) & $44(73.3)$ & $42(70.0)$ & NS \\
\hline Obesity (BMI >30), n (\%) & $22(36.7)$ & $24(40.0)$ & NS \\
\hline Smoking, n (\%) & $36(60.0)$ & $42(70.0)$ & NS \\
\hline Priority of surgery, n (\%) & & & 0.014 \\
\hline
\end{tabular}

Categorical variables are presented as the numbers $(n)$ and percentages (\%) and continuous variables as means \pm standard deviation. BMI, body mass index; NYHA, New York Heart Association functional class.

saline (PBS) (pH 7.4), dehydrated in an ascending series of alcohols, and embedded in paraffin via xylene. Fivemicrometre serial transverse sections were then cut and processed for standard histological (Mallory's trichromatic method, haematoxylin and eosin, resorcin and fuchsin) or immunohistochemical studies. For immunohistochemistry, endogenous peroxidase activity was quenched with $0.5 \%$ $\mathrm{H}_{2} \mathrm{O}_{2}$ in absolute methanol for 30 minutes at RT; slides were then permeabilized with $0.2 \%$ Triton $\mathrm{X}-100$ in PBS (20 min at RT) and incubated with $3 \%$ normal goat serum in PBS ( $30 \mathrm{~min}$ at $\mathrm{RT}$ ) to block nonspecific binding. Sections were then incubated overnight at $4{ }^{\circ} \mathrm{C}$ with primary polyclonal antibody to a specific markers of endothelial cells used to assess the integrity of the endothelial layer after surgical preparation. The following antibodies were used:
* CD31 (Dako 1:40) for assessment of endothelial morphological integrity;

* eNOS-enzyme eNOS (BD Biosciences 1:2500) for the endothelial functional integrity evaluation;

* CD133 (Novus Biologicals 1:3000), CD34 (Dako 1:50) for stratification of the regeneration potential.

Control sections were treated with normal rabbit immunoglobulin's at the same concentrations as for primary antibodies. The reaction was revealed by standard avidin-biotin peroxidase complex procedure using 3,3' diaminobenzidine (DAB) as chromogen. Images from immunostained sections were captured using a MIRAX MIDI Scanner and MIRAX ViewerSony (both Carl Zeiss, Jena, Germany).

For the assessment of CD133 antigen endothelial 
expression only qualitative analysis was carried out, where 1 indicated its endothelial expression and 0 no expression, respectively. For evaluation of other endothelial proteins, a method proposed by Hwang and colleagues was adopted (15). Tissue expression of CD31, CD34 and eNOS was graded semiquantitatively from 1 to 10 , based on the percentage of positive staining of the lumen length (e.g., 1 means $0 \%$ to $10 \%$ of luminal length, 2 means $10 \%$ to $20 \%$ of luminal length, etc.).

Microscopic analyse were performed by two researchers the most experienced in immunohistochemical assessment and blinded to the surgical method of LITA harvesting.

\section{Data presentation and statistical analysis}

First, the quantitative variables were checked for normality with the use of the Shapiro-Wilk W test. If they satisfied normal distribution criteria, they were expressed as the means \pm standard deviation. The variables without normal distribution were presented as the medians with the range (minimum-maximum). The categorical variables were expressed as the numbers (n) with percentages (\%). For statistical analysis Student's $t$ test or nonparametric alternatives such as Kolmogorov-Smirnov and $\chi^{2}$ tests were used as appropriate. A P value less than 0.05 was considered as significant. The analysis was performed using the Statistica ver. 10.0 software (StatSoft, Inc., Poland).

\section{Results}

All harvested mammary arteries were judged of acceptable outflow and quality to be used for myocardial revascularization.

Histological analysis of harvested LITA specimen demonstrated no major arterial damage such as wall disruption, dissection, thrombosis or subadventitial hematoma either in group $\mathrm{P}$ or group $\mathrm{S}$. Thus, no differences between groups were noted.

Tissue expression of CD133, CD34, and nitric oxide synthase eNOS (Figure 1) in the endothelial cells of the arterial segments in immunohistochemical assessment was comparable between both groups. It must be stressed that in $20 \%$ to $30 \%$ of studied LITA segments no expression of the aforementioned proteins was observed irrespective of applied surgical technique to harvest ITA. CD133 expression was noted in 43 (71.7\%) LITA cross sections in group $\mathrm{P}$ and in 41 (68.3\%) in group $\mathrm{S}$. The detailed results of endothelial immunoreactivity of other proteins are outlined in the Table 2 and in Figure 2.

Regarding CD31 tissue expression in the tunica intima, a qualitative assessment did not reveal differences between studied group. In $23 \%$ ( $n=14$ both in group $\mathrm{P}$ and group $\mathrm{S}$ ) of LITA specimens no expression of CD31 antigen was noted. However, statistical significance was reached $(\mathrm{P}<0.001)$ if semiquantitative analysis of endothelial expression was taken into consideration. Overall, it was markedly stronger in group P. Additionally, in group S, irregular areas with CD31 immunonegative fragments of endothelium, irrespective of expression grade, were also observed (Figure 3).

\section{Discussion}

One of the most crucial part of CABG procedures is harvesting of the autologous vessels that are to be applied as aorto-coronary bypass grafts. Unintentionally injury to these vascular conduits may result in both early and late graft failure (16). Macroscopic wall damage may be noticed by surgeons and such vessels must be disqualified from further usage. On the one side, skeletonization of LITA is linked to higher risk of macroscopic wall injury but on the other side it enables to identify such places. This assessment makes possible to disqualify such graft enough early to replace it with another one. Such strategy impacts favourably on long-term CABG outcomes. Surrounding tissues of the pedicled grafts although may be protective for artery during its harvesting but also make intraoperative macroscopic inspection almost impossible.

There are conflicting findings of the studies comparing two methods of LITA harvesting but performed on the dog experimental models. Daly et al. showed that skeletonization was associated with a significant decrease level of graft patency (to $67 \%$ ) compared to pedicled technique and that it was resulted predominantly from intimal hyperplasia (17). Del Campo hypothesized that the loss of natural resistance of LITA for hyperplastic changes and atherosclerosis might be associated with loss of vasa vasorum and functional paracrine activities of surrounding tissue and lymphatic edema vessels caused by the venous drainage loss, removed during the preparation (16). On the other hand, Sasajima et al., also in a dog model, noted at 3 and 12 weeks after initial surgical procedure no evidences of damage to the skeletonized artery. The endothelial layer remained intact, without signs of hypertrophy, necrosis, fibrosis and extracellular collagen fibres growth (18). Skeletonized artery was finely incorporated in the surrounding connective tissue 

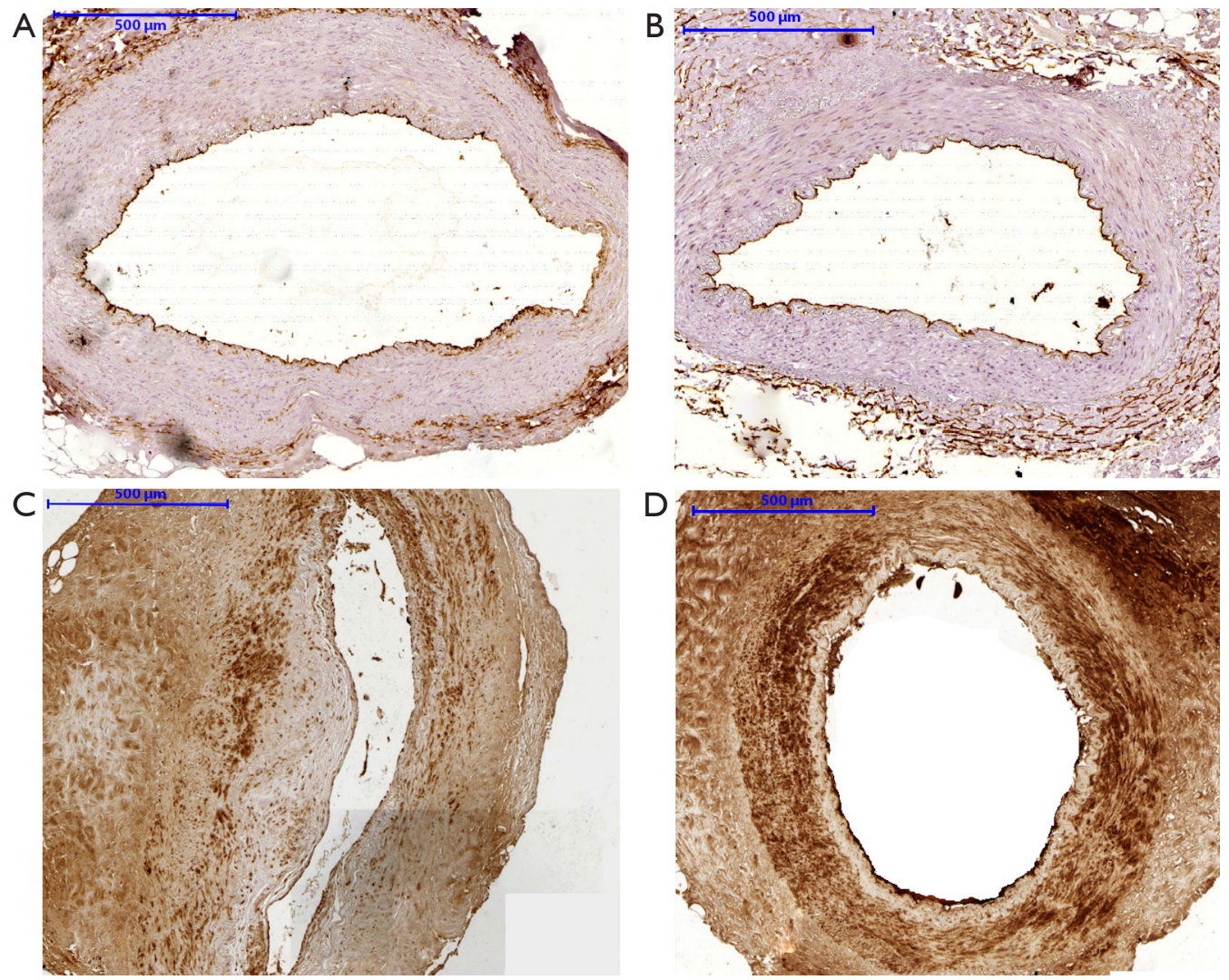

Figure 1 Immunohistochemical assessment of eNOS, CD34 expression. Endothelial tissue expression of CD34 (A,B) and eNOS (C,D) were comparable between groups. Semiquantitatively estimated CD34 endothelial expression was graded 8 and 9 in ITA segments harvested from (A) 54-year-old male using skeletonized (group S) and (B) 64-year-old man applying pedicled (group P) methods, respectively. Similarly, eNOS endothelial immunoreactivity was graded 8 in both ITA section. Segment C was obtained from 66-year-old female (group P) whereas segment D from 70-year-old man (group P). eNOS, endothelial nitric oxide synthase; ITA, internal thoracic artery.

Table 2 Endothelial expression of CD34, CD31 and eNOS

\begin{tabular}{llll}
\hline Studied protein & Group P & Group S & P value \\
\hline CD31 & $8.6 \pm 0.7$ & $7.8 \pm 1.0$ & $0.001^{\#}$ \\
CD34 & $8.8 \pm 0.9$ & $8.9 \pm 0.9$ & 0.690 \\
eNOS & $8.9 \pm 0.7$ & $9.1 \pm 0.8$ & 0.485 \\
\hline
\end{tabular}

Data are expressed as the mean with standard deviation. ", statistically significant. eNOS, endothelial nitric oxide synthase.

in which a number of microvessels had been developed. Ueda $e t a l$. in a similar experimental setting proved that the loss of vasa vasorum during skeletonization did not cause ischemia and then medial remodelling, proliferation of medial smooth muscle cells and eventually neointimal hyperplasia (19). Immunohistochemical assessment of proteins' expression, proved similar high activity of von
Willebrand factor (vWF) and nitric oxide synthase eNOS in vessels obtained either as skeletonized or pedicled grafts. Thus, preservation of structural and functional endothelial continuity was confirmed.

Several studies have been also carried out on the segments of LITA harvested intraoperatively from human beings. Noera et al. showed no endothelial hyperplasia and 
CD31

[n]

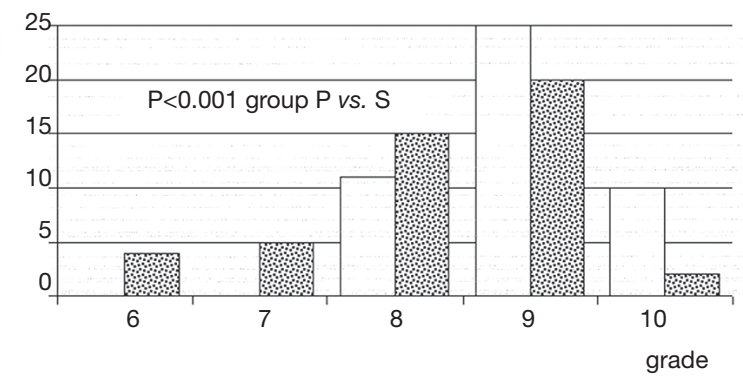

CD34

[n]

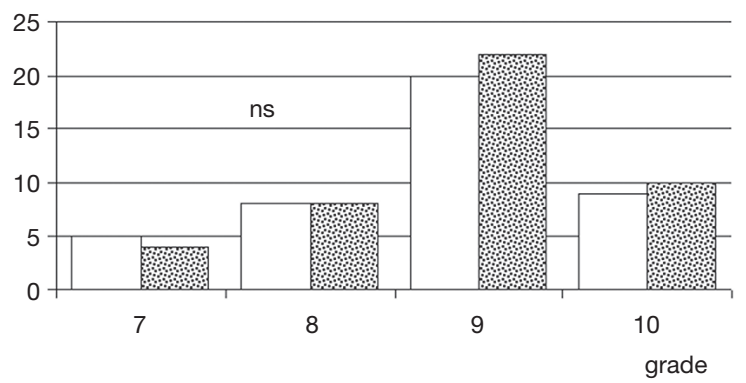

eNOS

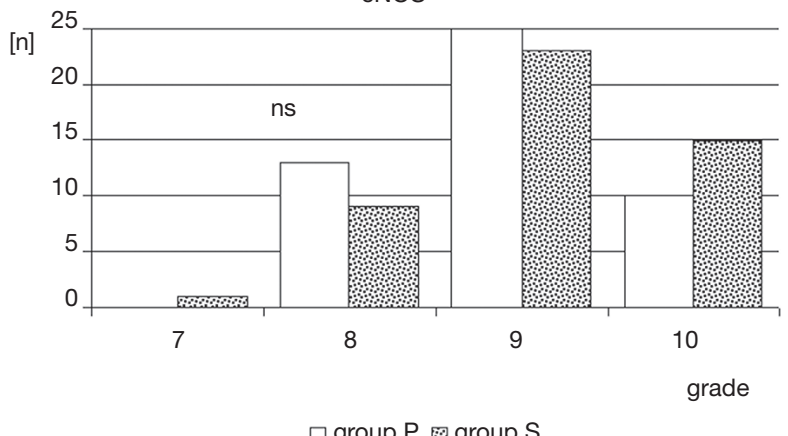

Figure 2 Detailed grading of endothelial expressions of CD31, CD34 and eNOS. Significant difference was found only in detailed analysis of endothelial CD31 immunoreactivity while expressions of CD34 and eNOS in the tunica intima of ITA were comparable. eNOS, endothelial nitric oxide synthase; ITA, internal thoracic artery.

microscopic dissections and lamina elastic damage under light microscopy (20). In a randomized trial, Gaudino et al. observed no macroscopic damage of arterial walls whereas two single cases of subadventitial hematoma, one in each group (skeletonized and pedicled, respectively) (21). Immunohistochemical staining showed a high degree of integrity of the endothelium $(97.2 \% \pm 1.9 \%$ in the skeletonized and $96.8 \% \pm 2.1 \%$ in pedicled group, respectively). In another clinical study, Yoshikai et al. confirmed in the electron microscopic study that structural integrity of LITA was completely dependent on safety of surgical manipulation (22). Opponents of skeletonization claimed it caused the arterial denervation and impaired vessel wall autoregulation. However, they failed to show it had any negative impact on the long-term outcomes. Deja et al. demonstrated increased sensitivity to norepinephrine and endothelium-independent relaxation to sodium nitroprusside in skeletonized samples of LITA (23). Malinowski et al. confirmed perivascular adipose tissue of LITA released potent nitric oxide- and prostacyclinindependent anticontractile factor, but without any clinical implications (24).

Our histological examination with classical staining techniques showed that the arterial segments were intact irrespective of harvesting method. We must stress again that all of them were harvested by very experienced cardiac surgeon. Moreover, we presume that grafts for scientific purposes are also harvested by the experienced surgeons that does not reflect daily cardiac surgical practice. Usually, residents or registrars are responsible for graft preparation.

In spite of the aforementioned facts, some differences regarding preservation of endothelial integrity between our groups have been noted in more detailed immunohistochemical assessment. In our study the preserved functional integrity of the intima evaluated by expression of eNOS was demonstrated in the vast majority of the arterial samples in both groups. eNOS synthase is responsible for the production of nitric oxide (NO), a potent factor promoting vascular muscle relaxation $(11,25)$. This result may indicate a similar functional potential to the relaxation of arteries that is of importance to sustain appropriate flow through aorto-coronary graft. Some authors tried to find association between skeletonizationrelated destruction of vasa vasorum and functional integrity. Kandemir et al. showed a significant reduction in the vasa vasorum accompanied by the lack of eNOS expression in skeletonized grafts (26). The others did not confirm that the concentration of vasa vasorum in the internal thoracic artery had any effect on the functional integrity of the vessels and long term clinical results (27).

In this study, authors for the first time analysed morphological integrity of LITA on the base of CD31 tissue expression. CD31 is a transmembrane protein located in the cells of the tunica intima, predominantly in the vascular endothelium. Previously, the morphological integrity of the arteries was assessed exclusively on the base of $\mathrm{vWF}$ expression. Usefulness of immunohistochemical 

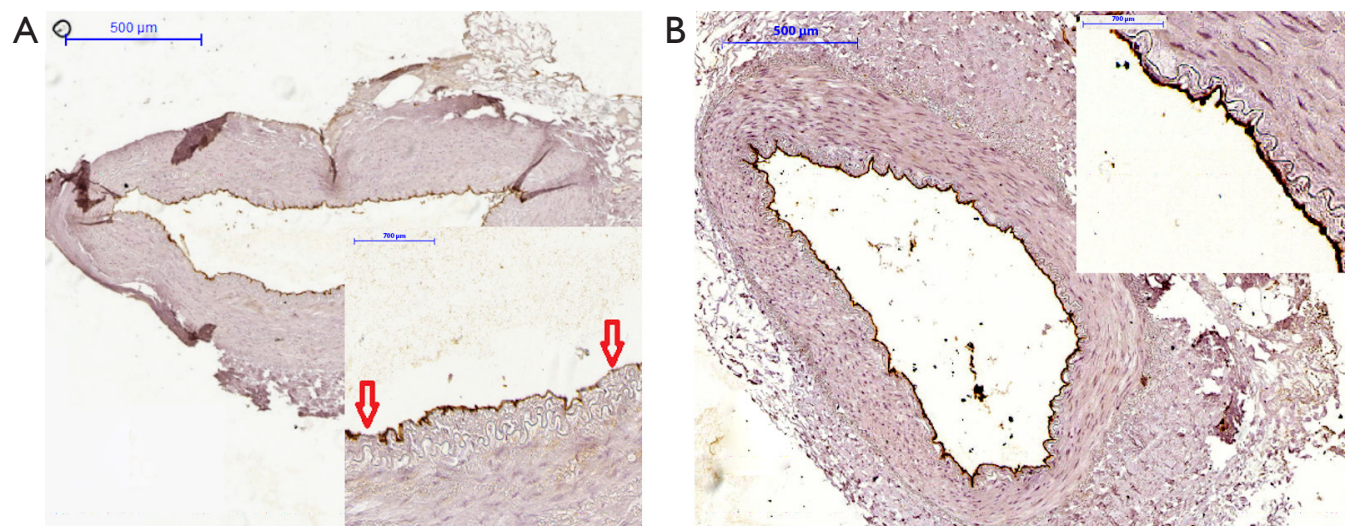

Figure 3 Immunohistochemical evaluation of endothelial CD31 expression. Immunohistochemical staining for CD31 showed markedly worse preservation of endothelium in group S (grade 4) than in group P (grade 10). Additionally, irregular areas with CD31 immunonegative fragments of the endothelium were observed particularly in group $\mathrm{S}$ (red arrows).

analysis of CD31 has been documented in the literature on the other vessels (12). Although, qualitative assessment of CD31 antigen expression did not reveal any differences between studied groups, more detailed analysis taking into consideration intensity of expression showed better preservation of morphological integrity in group P. It may indicate on higher degree of damage of the structural endothelium integrity and vascular injury during skeletonization. Irregular and weaker tissue expression may also result from artery uncontrolled expansion following loss of support of the surrounding tissues. It has already known that skeletonized arteries had usually slightly bigger diameter as compared to arteries in the pedicle.

Moreover, a novel approach, applied by our team, to study an impact of harvesting technique on preservation of vascular endothelium function included also an estimation of potential for vascular repair and restoration of endothelial function. Thus, endothelial expression of additional proteins such as CD34 and CD133 was also studied. EPCs demonstrating their expression released from bone marrow play an important role in post-natal neoangiogenesis, neovascularization and repair processes in damaged intima. CD133 is a marker for immature progenitor cells and CD34 can provide a high proliferative potential to cell colony formation. It was shown previously the decrease of EPC numbers and activity may contribute to impaired vascularization in patients with coronary artery disease CAD (28). Moreover, intracoronary transfusion of circulation-derived $\mathrm{CD} 34^{+}$cells was found to improved left ventricular function in patients with end-stage diffuse CAD unsuitable for coronary interventions (29). Our analysis showed comparable endothelial regeneration potential in both groups. It may suggest that despite of higher risk of skeletonization-related impairment in endothelial morphological integrity, preserved recovery mechanisms will be sufficient to ensure its continuity during postoperative course and clinically without unfavorable impact on the long term LITA patency.

We are aware of some limitations of our study. Our analysis involved although consecutive but still a limited number of CABG patients. Including more subjects, it would likely be possible to link histological findings to some clinical variables including systemic diseases such as arterial hypertension, hyperlipidemia or diabetes mellitus. Additionally, immunohistochemical analysis of proteins tissue expression may be subjected to observerrelated bias. Having it in mind, histologists experienced in immunohistochemistry were invited to participate in this study. Moreover, aforementioned fact that all LITA conduits were harvested by experienced consultant does not reflect daily clinical practice in the cardiac surgery departments. It is possible that residents would cause more trauma to the harvested LITA grafts, particularly when applying technique of skeletonization. Eventually, the value of our findings should be validated by the clinical longterm results. However, it would be very difficult to find any clinical differences since the rate of LITA failure is usually below 10\% during 20-year follow-up period. 


\section{Conclusions}

The skeletonized method of LITA harvesting may be associated with slightly worse preservation of morphological integrity of endothelium but without compromising functional integrity and potential for tissue regeneration.

\section{Acknowledgements}

This study was supported by the authors' institution.

\section{Footnote}

Conflicts of Interest: The authors have no conflicts of interest to declare.

Ethical Statement: The study protocol was approved by the local bioethical committee (No. 32.04.2017.IRB) and all study participants expressed informed consent to randomization and specimen collection.

\section{References}

1. He GW. Arterial grafts for coronary artery bypass grafting: biological characteristics, functional classification, and clinical choice. Ann Thorac Surg 1999;67:277-84.

2. Loop FD. Internal-thoracic-artery grafts. Biologically better coronary arteries. N Engl J Med 1996;334:263-5.

3. Barner HB, Barnett MG. Fifteen- to twenty-one-year angiographic assessment of internal thoracic artery as a bypass conduit. Ann Thorac Surg 1994;57:1526-8.

4. Benedetto U, Altman DG, Gerry S, et al. Pedicled and skeletonized single and bilateral internal thoracic artery grafts and the incidence of sternal wound complications: Insights from the Arterial Revascularization Trial. J Thorac Cardiovasc Surg 2016;152:270-6.

5. Ali E, Saso S, Ashrafian H, et al. Does a skeletonized or pedicled left internal thoracic artery give the best graft patency? Interact Cardiovasc Thorac Surg 2010;10:97-104.

6. Taggart DP. Current status of arterial grafts for coronary artery bypass grafting. Ann Cardiothorac Surg 2013;2:427-30.

7. Peterson MD, Borger MA, Rao V, et al. Skeletonization of bilateral internal thoracic artery grafts lowers the risk of sternal infection in patients with diabetes. J Thorac Cardiovasc Surg 2003;126:1314-9.

8. Cunningham JM, Gharavi, MA, Fardin R, et al. Considerations in the skeletonization technique of internal thoracic artery dissection. Ann Thorac Surg 1992;54:94750; discussion 951.

9. Sepehripour AH, Jarral OA, Shipolini AR, et al. Does a 'no-touch' technique result in better vein patency? Interact Cardiovasc Thorac Surg 2011;13:626-30.

10. Yacoub MH. Reply to Cunningham JM. Skeletonization of internal thoracic artery grafts. J Thorac Cardiovasc Surg 1996;111:489-90.

11. Nowicki M, Buczkowski P, Miskowiak B, et al. Immunocytochemical study on endothelial integrity of saphenous vein grafts harvested by minimally invasive surgery with the use of vascular mayo stripers. A randomized controlled trial. Eur J Vasc Endovasc Surg 2004;27:244-50.

12. Nowicki M, Misterski M, Malinska A, et al. Endothelial integrity of radial artery grafts harvested by minimally invasive surgery - immunohistochemical studies of CD31 and endothelial nitric oxide synthase expressions: a randomized controlled trial. Eur J Cardiothorac Surg 2011;39:471-7.

13. Gansera B, Rohrbach $\mathrm{H}$, Gillrath $\mathrm{G}$, et al. Is there a difference between diabetic and non-diabetic ITAs? Histomorphological and Immunohistochemical examinations of Internal Thoracic Arteries. Thorac Cardiovasc Surg 2004;52:255-60.

14. Hilker M, Buerke M, Lehr HA, et al. Bypass graft disease: analysis of proliferative activity in human aorto-coronary bypass grafts. Heart Surg Forum 2002;5:S331-41.

15. Hwang HY, Kim MA, Seo JW, et al. Endothelial preservation of the minimally manipulated saphenous vein composite graft: histologic and immunohistochemical study. J Thorac Cardiovasc Surg 2012;144:690-6.

16. Del Campo C. Pedicled or skeletonized? A review of the internal thoracic artery graft. Tex Heart Inst J 2003;30:170-5.

17. Daly RC, McCarthy PM, Orszulak TA, et al. Histologic comparison of experimental coronary artery bypass grafts. Similarity of in situ and free internal mammary artery grafts. J Thorac Cardiovasc Surg 1988;96:19-29.

18. Sasajima T, Wu MH, Shi Q, et al. Effect of skeletonizing dissection on the internal thoracic artery. Ann Thorac Surg 1998;65:1009-13.

19. Ueda T, Taniguchi S, Kawata T, et al. Does skeletonization compromise the integrity of internal thoracic artery grafts? Ann Thorac Surg 2003;75:1429-33.

20. Noera G, Pensa P, Lodi R, et al. Influence of different harvesting techniques on the arterial wall of the internal mammary artery graft: microscopic analysis. Thorac 
Cardiovasc Surg 1993;41:16-20.

21. Gaudino M, Toesca A, Nori SL, et al. Effect of skeletonization of the internal thoracic artery on vessel wall integrity. Ann Thorac Surg 1999;68:1623-7.

22. Yoshikai M, Tsuyoshi I, Kamohara K, et al. Endothelial integrity of ultrasonically skeletonized internal thoracic artery: morphological analysis with scanning electron microscopy. Eur J Cardiothorac Surg 2004;25:208-11.

23. Deja MA, Golba KS, Malinowski M, et al. Skeletonization of internal thoracic artery affects its innervation and reactivity. Eur J Cardiothorac Surg 2005;28:551-7.

24. Malinowski M, Deja MA, Golba KS, et al. Perivascular tissue of internal thoracic artery releases potent nitric oxide and prostacyclin-independent anticontractile factor. Eur J Cardiothorac Surg 2008;33:225-31.

25. Broeders MAV, Doevendans PA, Maessen JG, et al. The human internal thoracic artery releases more nitric oxide in response to vascular endothelial growth factor than

Cite this article as: Puslecki M, Buczkowski P, Nowicki M, Sujka-Kordowska P, Ligowski M, Misterski M, Stefaniak S, Dąbrowski M, Dabrowska A, Bocianski M, Szarpak L, Ruetzler K, Jemielity M, Perek B. An innovative panel to assess endothelial integrity of pedicled and skeletonized internal thoracic artery used as aortocoronary bypass graft: a randomized comparative histologic and immunohistochemical study. J Thorac Dis 2018;10(8):4865-4873. doi: 10.21037/ jtd.2018.07.62 the human saphenous vein. J Thorac Cardiovasc Surg 2001;122:305-9.

26. Kandemir O, Buyukates M, Gun BD, et al. Intraoperative and histochemical comparison of the skeletonized and pedicled internal thoracic artery. Heart Surg Forum 2007;10:E158-61.

27. Athanasiou T, Crossman MC, Asimakopoulos G, et al. Should the internal thoracic artery be skeletonized? Ann Thorac Surg 2004;77:2238-46.

28. Vasa M, Fichtlscherer S, Aicher A, et al. Number and migratory activity of circulating endothelial progenitor cells inversely correlate with risk factors for coronary artery disease. Circ Res 2001;89:E1-7.

29. Lee FY, Chen YL, Sung PH, et al. Intracoronary Transfusion of Circulation-Derived CD34+Cells Improves Left Ventricular Function in Patients With End-Stage Diffuse Coronary Artery Disease Unsuitable for Coronary Intervention. Crit Care Med 2015;43:2117-32. 